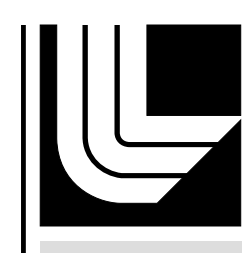

LAWRENCE LIVERMORE NATIONAL LABORATORY

\title{
Improved Optical Quality for Ti:Sapphire using MRF
}

K. I. Schaffers, C. P. J. Barty, A. J. Bayramian, J. A.

Caird, P. J. Davis, C. A. Ebbers, J. A. Menapace, D. B. Joyce, F. Schmid, K. Schmid

July 3,2007

HEC DPSSL Workshop

Osaka, Japan

September 17, 2007 through September 19, 2007 
This document was prepared as an account of work sponsored by an agency of the United States government. Neither the United States government nor Lawrence Livermore National Security, LLC, nor any of their employees makes any warranty, expressed or implied, or assumes any legal liability or responsibility for the accuracy, completeness, or usefulness of any information, apparatus, product, or process disclosed, or represents that its use would not infringe privately owned rights. Reference herein to any specific commercial product, process, or service by trade name, trademark, manufacturer, or otherwise does not necessarily constitute or imply its endorsement, recommendation, or favoring by the United States government or Lawrence Livermore National Security, LLC. The views and opinions of authors expressed herein do not necessarily state or reflect those of the United States government or Lawrence Livermore National Security, LLC, and shall not be used for advertising or product endorsement purposes. 


\title{
Improved Optical Quality for Ti:Sapphire using MRF
}

\author{
K.I. Schaffers, C. P. J. Barty, A.J. Bayramian, J. A. Caird, P. J. Davis, \\ C.A. Ebbers, and J. A. Menapace \\ Lawrence Livermore National Laboratory, 7000 East Ave., L-482, Livermore, CA 94550-9234 USA \\ Phone: (925)422-5084, FAX: (925)424-2495, email: schaffers1@llnl.gov \\ D. B. Joyce, F. Schmid, and K. Schmid \\ Crystal Systems, Inc., 27 Congress Street, Salem, MA 01970
}

\begin{abstract}
Magneto-rheological finishing (MRF) imprinting techniques have been applied to Ti:sapphire crystals to compensate for submillimeter distortions, thereby, improving the transmitted wavefront and increasing the availability of large aperture parts.
\end{abstract}

\section{Introduction}

Ti:sapphire has become the premier material for femtosecond high peak power laser systems because of its wide bandwidth ranging from 680 to $1100 \mathrm{~nm}$, peaking at $800 \mathrm{~nm}$. Extension to higher energies is limited by the size of the amplication medium. Yields of high quality large diameter crystals have been constrained by lattice distortions that may appear in the boule limiting the usable area from which high quality optics can be harvested. This is a complication for both clear sapphire and Ti:sapphire. In this paper we demonstrate for the first time that Magneto-rheological finishing (MRF) can be used to compensate for these lattice distortions in the transmitted wavefront of Ti:sapphire to produce high quality optics from otherwise unusable parts. This work may allow extension of Ti:sapphire based systems to peak powers well beyond one pettawatt.

\section{Ti:Sapphire crystals}

Ti:sapphire crystals are grown by the Heat Exchanger Method (HEM) at Crystal Systems, Inc. as shown in Figure 1(a). "In this method, the solid-liquid interface is submerged beneath the surface of the melt, resulting in uniform temperature gradients at the interface. This results in the high quality crystal perfection essential for efficient lasing."'11] Boule sizes of $15 \mathrm{~cm}$ diameter are currently being grown from which $10 \mathrm{~cm}$ diameter high quality optics can be harvested on a routine basis. Soon this technology will be pushed to $20 \mathrm{~cm}$ diameters to harvest even larger optics for innovative new laser designs. The limiting factor for harvesting of large diameter parts, are lattice distortions that can occur in boules which manifest themselves as refractive index changes. Distortions can vary from about 0.3-5 mm in width as shown in the transmitted wavefront in Figure 1(b). This distortion is large enough to disrupt the quality of a laser beam, which can cause damage to optics downstream in a laser system, and for short pulse systems can lead to incomplete compression and poor ability to focus the laser beam. As a result, any laser optic of Ti:sapphire that has these types of lattice distortions is less desirable for applications that require good transmission and beam quality.

\section{Magneto-Rheological Finishing (MRF)}

Magneto-rheological finishing (MRF) imprinting techniques have been developed as a proven method for compensating for lattice distortions and refractive index variations in glass and crystalline materials to provide for low transmitted wavefronts in plano-optics. [2] In particular, MRF has made a significant contribution in compensating for low angle grain boundaries in $\mathrm{Yb}: \mathrm{S}-\mathrm{FAP}\left[\mathrm{Yb}^{3+}: \mathrm{Sr}_{5}\left(\mathrm{PO}_{4}\right)_{3} \mathrm{~F}\right]$ crystals. We have chosen MRF to compensate 
for the submillimeter lattice distortions of sapphire and Ti:sapphire crystals to improve the transmitted wavefront. Currently, commercial MRF capabilities only compensate for long spatial period phase distortions of $3 \mathrm{~mm}$ or greater. LLNL provides the expertise and MRF equipment capability to correct for shorter period phase distortions that can be applied to unique crystalline materials. MRF machine improvements involve novel topographical gradient fitting routines, polishing protocols, and specialized operating conditions that can deterministically correct optical errors in the submillimeter range to improve the transmitted wavefront. Using the LLNL MRF technique it is possible to achieve low transmitted wavefronts in Ti:sapphire and sapphire crystals, which will increase the availability of larger high-precision optics for many stringent optical and laser applications. Preliminary Ti:sapphire parts have shown marked wavefront improvement.
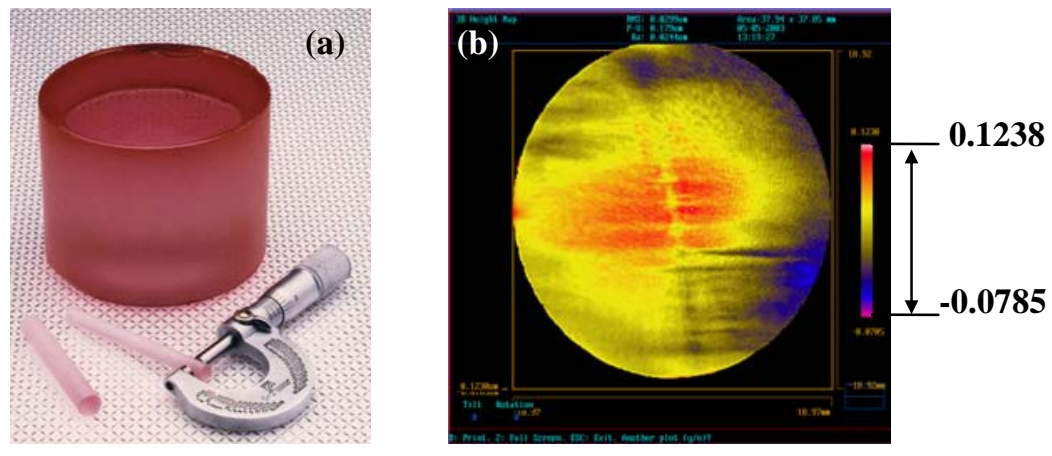

Figure 1. (a) High quality Ti:sapphire grown at Crystal Systems by HEM. (b) Transmitted wavefront of a lattice distortion that can appear in sapphire and Ti:sapphire crystals.

\section{Summary}

MRF has been proven to compensate for lattice distortions that can appear in sapphire and Ti:sapphire crystals as they are scaled to larger diameters. With this technology low transmitted wavefront errors can be achieved from otherwise undesirable material for short pulse laser operation. Compensation of any potential lattice distortions in both sapphire and Titanium-doped sapphire will increase the availability of larger high-precision optics for many stringent optical and laser applications.

This work was performed under the auspices of the U.S. Department of Energy by the University of California Lawrence Livermore National Laboratory under contract No. W-7405-Eng-48.

\section{References}

[1] W. R. Rapoport, C. P. Kattak, "Titanium sapphire laser characteristics," Appl. Opt. 27(13) 2677-2684 (1988). Also, a direct quote from the Crystal Systems website on Ti:sapphire.

[2] K. I. Schaffers, J. A. Menapace, et.al., "Growth of large laser crystals for high power lasers," Third International Workshop on Crystal Growth Technology, Beatenberg, Switzerland, (Sept. 2005). 